\title{
Analysis on Relevant Music Therapy Elements and Its Effectiveness on Treating AD
}

\author{
Yutian Zhang \\ College of Biological Science, University of California Davis, Davis, California, 95616, United States \\ ${ }^{*}$ Corresponding author. Email: gaoming@cas-harbour.org
}

\begin{abstract}
Alzheimer's disease (AD) is a serious but common health condition for old people, and it is different from normal aging. Finding an effective treatment and ultimate cure for $\mathrm{AD}$ has become a hot topic in the field of medicine and neuroscience nowadays. However, due to the complexity of the disease and characteristics of our neurons, identifying useful pharmacological treatments and implementing them into the clinial settings are difficult. Music therapy (MT), a nonpharmacological treatment, was proposed a long time ago and was believed to be useful for easing cognitive and mood symptoms of AD. Previous research has shown the rehabilitation effect of music on AD patients, but more detailed instruction on how to carry out music therapy and choose the right music pieces are needed. To contribute better in the music therapy field, this paper proposed two elements of music that therapists and researchers should consider: emotion and familiarity, and it also analyzed possible effects of different music therapeutic approaches on patients. Further investigations on how positive and negative emotional musics as well as familiar or unfamiliar music were needed due to deficiency of current research. Therapists should also consider finding more resultful strategies like individualized music treatment and combine MT with meditations.
\end{abstract}

Keywords: Alzheimer's disease; music therapy; emotion; meditation; familiarity

\section{INTRODUCTION}

Alzheimer's disease (AD) is a well-known neurodegenerative disease that affects millions of people worldwide. In the U.S, 1 out of 9 people aged older than 65 years was suffering from AD in 2021, and the prevalence rate is increasing continuously due to growing aging populations [1]. The main brain changes that cause $\mathrm{AD}$ in patients are the accumulation of amyloid-beta and tau protein tangles in neurons, which ultimately result in cell function loss and cell death [1]. Because brain areas that are involved in $\mathrm{AD}$ often include memory-associated hippocampus area and cerebral cortex, major characteristics of $\mathrm{AD}$ are progressive loss of memory and problems in cognition. Old people who suffer from $\mathrm{AD}$ in the late stages are usually not able to take care of themselves, and the ultimate result is fatal. Despite much hard work from researchers nowadays, only some treatments were available to ease symptoms of the disease. Finding ultimate pharmacological cures for $\mathrm{AD}$ is still in the progress. Therefore, music therapy, which has a long history, was brought up to the stage as a psychological intervention outside traditional pharmacological treatment and was believed to be a promising method for treating $\mathrm{AD}$ [2].

It has been presented by many papers in the past that listening to music has an enhancing effect on people's cognitive ability. Teppo Särkämö and his colleague [3] worked on cerebral artery stroke patients and found a significant increase in patients' verbal memory and attention after they were exposed to music every day. Another finding from Muireann Irish et.al proved that AD patients who in the background music group showed significantly better autobiographical memory recall, along with a reduction in anxiety level, than those in the control group [4]. Taking multiple findings together, music has its potential to be used for releasing mood symptoms and treating cognitive impairment in healthcare or clinical environments. The extent to which a patient can recover their memory and cognitive ability, however, can vary depending on the specific characteristic of the music cue. In this paper, emotion and familiarity were discussed along with their effect on patients' cognitive ability recovery. 


\section{ELEMENTS IN A MUSIC}

To implement music therapy at its maximum benefits, choosing the right pieces of music as the stimulation material is important. The following two sections will discuss two aspect of music elements: emotion and familiarity.

\subsection{Music with emotions}

It is well-established that emotion plays an important role in mediating human learning and memory. Extensive research and functional magnetic resonance imaging studies have shown that there's a connection between the emotion-regulating area amygdala and memory-related area of the hippocampus, in the view that the basolateral region of the amygdala modulates memory consolidation through talking with the hippocampus [5]. This role of amygdala in memory formation is largely through influencing a process called long-term potentiation (LTP), a mechanism that was believed to induce memory formation [6]. Animal experimental study illustrated that basolateral amygdala stimulation activates early LTP in hippocampus [7]. Interestingly, alteration and dysfunction of LTP have been found in AD patients [8], suggesting that implementing emotional-inducing stimulus may help restore the normal function of LTP in AD patients and improve their memory functions.

Continuing with this view, understanding the emotional aspect of music is important when it comes to improving the effectiveness of music therapy. It's necessary to investigate how music with positive and negative emotions influences memory recall in AD patients. There's a belief that music that is presented as "sad" serves an adaptive purpose to help evoke remote autobiographical memory in AD patients, whereas positive memory does not retain this function [9]. Taking this view, Juan and other colleagues replicated a study from Foster and Valentine with additional emotional aspects of music tested $[9,10]$. Except proving that emotion-evoking music improved autobiographical memory recall in AD patients better than non-emotion presenting music, they also showed that sad music enhanced memory recovery better than happy music, with a stronger effect specifically on semantic remote memory [9]. This result could be partially explained by another study, where researchers applied fMRI techniques to investigate sad and happy music in the context of healthy participants [11]. In their experiments, they illustrated that sad music evokes stronger mindwandering (which is an internally oriented self-reflection process), and participants who listened to sad music shifted their attention inward with a better selfrecognition than those who listened to happy music $[11,12]$. When engaging in sad music listening, people tend to focus more on themselves rather than music per se. This could be one possible explanation of why sad music serves as a stronger external cue for memory recall in $\mathrm{AD}$ patients. However, due to the insufficiency of current studies, the exact differentiation between effects of sad and happy music and their mechanisms are still in need of further investigation.

\subsection{Familiarity of music}

Familiarity, another dimension of music, can potentially influence the effectiveness of music therapy. Difference between familiar and unfamiliar music was observed in an EEG study, where familiar music evokes distinct brain changes and more pupil dilation in human than unfamiliar music [13]. Similarly, a fMRI study investigated the interaction between familiarity and emotion, found that even though musical preference does not play a role in brain changes, a significant increase in blood oxygen level dependence (BOLD) index was observed in emotion-relevant area while listening to familiar music, which indicates that familiar music stimulates people's emotion more strongly than unfamiliar music [14].

However, despite much significance of familiarity in music listening was identified, past literature presented contradictory results. Foster and Valentine did an experiment on dementia participants with either novel or familiar music, aiming to investigate the influence of background music on their autobiographical memory recall. They found no difference in terms of the familiarity level of music, though this result was not directly measured [15]. Later experiment, also done by Foster and Valentine, confirmed again that there's no effect between familiar music and novel music [10]. Taking both results from the image studies and experimental studies, the actual effect of familiarity of music listening on memory recall was unclear. Interestingly, the effect of familiarity on selfconsciousness was proved in another study [16]. After familiar and novel music intervention, AD patients in familiar music groups showed an improvement in their self-consciousness, whereas a degeneration in selfconsciousness was observed for patients treated with unfamiliar music [16]. Since they did not measure participant's performance on memory recall, it is unclear whether familiarity plays a role in memory-related events. Despite the fact that familiarity is an essential element in music, current literature researching on this topic were rare, even available studies produce heterogeneous results. Further investigations are required in the future to better understand whether familiar music differs from novel music so therapists can take advantage of these results. 


\section{DIFFERENT MUSIC INTERVENTION APPROACHES}

In addition to emotions and familiarity, the way of implementing music therapy could also influence the outcome.

Besides letting patients listen to music alone, leading them to actively participate in music like singing along with therapists or dancing could produce different results. Mayumi Sakamoto and his colleagues compared passive and interactive music intervention approaches in severe AD patients [17]. They found out that letting participants engage into the music through multiple interactive activities like singing, clapping, and dancing led to stronger reduction in both the psychological and behavioral symptoms of dementia [17]. On the contrary, a study from Tsoi and other researchers claimed that giving passive music stimulation was more effective in reducing behavioral and psychological agitation problems than guiding participants to actively participating in music [18]. Results from current literatures did not reach accordance with each other, therefore, further evidence was needed to compare the effect of interactive music and passive music approach in clinical settings.

Mindfulness meditation, an approach usually used for treating anxiety disorders, was proved to be useful for $\mathrm{AD}$ and dementia. Increase in overall quality and satisfaction of life, enhancement in mood and stress, and reduction in the level of cognitive and behavioral deterioration were all observed in several literatures [19]. Considering similar effects of music, it is interesting to compare the influence of music therapy and meditation. Innes and other researchers did experiments on old adults with subjective cognitive decline and showed that meditation, specifically Kartan Kriya meditation, improves mood and mental wellness more prominently than listening to music alone [20]. Similarly, in other experiments done by the same authors, they confirmed several biomarkers related to cognitive and mood symptom improvement and corroborated the mood rehealing effect of meditation [21]. Nonetheless, since none of these two researchers explore the effect on autobiographical memory recovery specifically, it is unclear whether meditation could be used as a stronger approach for memory restore. What we can confirm now is the healing effect of meditation on emotional stress and anxiety.

In fact, most of the meditation procedures involve music intervention, where people usually perform meditation activities while playing certain music or sound. Soft music in the background intends to help participants concentrate better on their internal reflection and block out noises or uneasy thoughts from the outside environment. Thus, implementing meditation activity into music therapy when treating AD patients may have stronger impacts in helping them to reduce psychological stress and improve wellbeing besides benefits from music alone.

\section{CONCLUSION}

Research in the field have proved the rehabilitating effect of music in multiple aspects like recovery of autobiographical memory, behavioral symptoms, and mood symptoms. Two elements of music should be considered: emotion and familiarity. Emotional music can produce stronger healing effects generally, but the exact differentiation between sad and happy emotional music is unclear. Also, understanding the effect of familiarity is important for successful music therapy approaches. Finally, combining music therapy with meditation is recommended in future therapeutic situations. To improve MT and better serve patients, more randomized, blinded, and clinically done experiments are necessary. Since AD is a multifaceted disease, however, mind-body therapies like MT should only be used as a complementary approach to treat AD. Researchers should continue working on inventing pharmacological treatments.

\section{REFERENCES}

[1] 2021 Alzheimer's disease facts and figures. (2021). Alzheimer's \& dementia : the journal of the Alzheimer's Association, 17(3), 327-406. https://doi.org/10.1002/alz.12328

[2] Kayaaslan B, Lok N. (2019) The Effect of Music Therapy on Cognitive Functions and Adaptation in Alzheimer's Patients. Int J Depress Anxiety, 2:014. doi.org/10.23937/2643-4059/1710014

[3] Särkämö, T., Tervaniemi, M., Laitinen, S., Forsblom, A., Soinila, S., Mikkonen, M., Autti, T., Silvennoinen, H. M., Erkkilä, J., Laine, M., Peretz, I., \& Hietanen, M. (2008). Music listening enhances cognitive recovery and mood after middle cerebral artery stroke. Brain : a journal of neurology, 131(Pt 3), 866-876. https://doi.org/10.1093/brain/awn013

[4] Irish, M., Cunningham, C. J., Walsh, J. B., Coakley, D., Lawlor, B. A., Robertson, I. H., \& Coen, R. F. (2006). Investigating the enhancing effect of music on autobiographical memory in mild Alzheimer's disease. Dementia and geriatric cognitive disorders, 22(1), 108-120. https://doi.org/10.1159/000093487

[5] McGaugh, J.D. (2002). Memory consolidation and the amygdala: a systems perspective. Trends in Neurosciences, 25, 456-461.

[6] Maren S. (1999). Long-term potentiation in the amygdala: a mechanism for emotional learning and memory. Trends in neurosciences, 22(12), 561-567. https://doi.org/10.1016/s0166-2236(99)01465-4 
[7] Frey, S., Bergado-Rosado, J., Seidenbecher, T., Pape, H. C., \& Frey, J. U. (2001). Reinforcement of early long-term potentiation (early-LTP) in dentate gyrus by stimulation of the basolateral amygdala: heterosynaptic induction mechanisms of late-LTP. The Journal of neuroscience : the official journal of the Society for Neuroscience, 21(10), 3697-3703. https://doi.org/10.1523/JNEUROSCI.21-1003697.2001

[8] Mango, D., Saidi, A., Cisale, G. Y., Feligioni, M., Corbo, M., \& Nisticò, R. (2019). Targeting Synaptic Plasticity in Experimental Models of Alzheimer's Disease. Frontiers in pharmacology, 10, 778. https://doi.org/10.3389/fphar.2019.00778

[9] Meilán García, J. J., Iodice, R., Carro, J., Sánchez, J. A., Palmero, F., \& Mateos, A. M. (2012). Improvement of autobiographic memory recovery by means of sad music in Alzheimer's Disease type dementia. Aging clinical and experimental research, 24(3), 227-232. https://doi.org/10.3275/7874

[10] Foster, N. A., \& Valentine, E. R. (2001). The effect of auditory stimulation on autobiographical recall in dementia. Experimental aging research, 27(3), 215228. https://doi.org/10.1080/036107301300208664

[11] Taruffi, L., Pehrs, C., Skouras, S. et al. Effects of Sad and Happy Music on Mind-Wandering and the Default Mode Network. Sci Rep 7, 14396 (2017). https://doi.org/10.1038/s41598-017-14849-0

[12] Dixon, M. L., Fox, K. C., \& Christoff, K. (2014). A framework for understanding the relationship between externally and internally directed cognition. Neuropsychologia, $\quad 62, \quad 321-330$. https://doi.org/10.1016/j.neuropsychologia.2014.05 .024

[13] Jagiello, R., Pomper, U., Yoneya, M. et al. Rapid Brain Responses to Familiar vs. Unfamiliar Music an EEG and Pupillometry study. Sci Rep 9, 15570 (2019). https://doi.org/10.1038/s41598-019-517599

[14] Pereira, C. S., Teixeira, J., Figueiredo, P., Xavier, J., Castro, S. L., \& Brattico, E. (2011). Music and emotions in the brain: familiarity matters. PloS one, $6(11)$, e27241. https://doi.org/10.1371/journal.pone.0027241

[15] Foster, N. A., \& Valentine, E. R. (2001). The effect of auditory stimulation on autobiographical recall in dementia. Experimental aging research, 27(3), 215228. https://doi.org/10.1080/036107301300208664

[16] Arroyo-Anlló, E. M., Díaz, J. P., \& Gil, R. (2013). Familiar music as an enhancer of self-consciousness in patients with Alzheimer's disease. BioMed research international, 2013, 752965. https://doi.org/10.1155/2013/752965

[17] Sakamoto, M., Ando, H., \& Tsutou, A. (2013). Comparing the effects of different individualized music interventions for elderly individuals with severe dementia. International psychogeriatrics, 25(5), $775-784$. https://doi.org/10.1017/S1041610212002256

[18] Tsoi, K., Chan, J., Ng, Y. M., Lee, M., Kwok, T., \& Wong, S. (2018). Receptive Music Therapy Is More Effective than Interactive Music Therapy to Relieve Behavioral and Psychological Symptoms of Dementia: A Systematic Review and Meta-Analysis. Journal of the American Medical Directors Association, $\quad$ 19(7), 568-576.e3. https://doi.org/10.1016/j.jamda.2017.12.009

[19] Russell-Williams, J., Jaroudi, W., Perich, T., Hoscheidt, S., El Haj, M., \& Moustafa, A. A. (2018). Mindfulness and meditation: treating cognitive impairment and reducing stress in dementia. Reviews in the neurosciences, 29(7), 791-804. https://doi.org/10.1515/revneuro-2017-0066

[20] Innes, K. E., Selfe, T. K., Khalsa, D. S., \& Kandati, S. (2016). Effects of Meditation versus Music Listening on Perceived Stress, Mood, Sleep, and Quality of Life in Adults with Early Memory Loss: A Pilot Randomized Controlled Trial. Journal of Alzheimer's disease : JAD, 52(4), 1277-1298. https://doi.org/10.3233/JAD-151106

[21] Innes KE, Selfe TK, Brundage K, Montgomery C, Wen S, Kandati S, Bowles H, Khalsa DS, Huysmans Z. Effects of Meditation and Music-Listening on Blood Biomarkers of Cellular Aging and Alzheimer's Disease in Adults with Subjective Cognitive Decline: An Exploratory Randomized Clinical Trial. J Alzheimers Dis. 2018;66(3):947970. doi: 10.3233/JAD-180164. PMID: 30320574; PMCID: PMC6388631. 